\title{
On Non-Supersymmetric String Model Building
}

\author{
John Rizos ${ }^{* \dagger}$ \\ University of Ioannina \\ E-mail: irizosduoi.gr
}

We study a class of heterotic string compactifications possessing $S O(10)$ "observable" gauge symmetry and spontaneously broken supersymmetry via the Scherk-Schwartz mechanism. We formulate a set of phenomenological criteria and identify models that comply with all requirements. This approach allows us to address and propose solutions to some of the open problems in non-supersymmetric string model building including the vacuum instability due to the appearance of tachyons and dilaton tadpoles, the presence of chiral fermions and the existence of Standard Model breaking light Higgs fields.

Corfu Summer Institute 2019 "School and Workshops on Elementary Particle Physics and Gravity" (CORFU2019)

31 August - 25 September 2019

Corfü, Greece

*Speaker.

${ }^{\dagger}$ A footnote may follow. 


\section{Introduction}

Space-time supersymmetry (SUSY) is one of the most compelling extensions of the Standard Model (SM) of particle interactions. In the context of SUSY, a (yet-to-be-discovered) supersymmetric partner is associated to every SM particle. SUSY can account for hierarchy and gauge coupling unification and provide a natural candidate for dark matter. However, as of today, experiments have not provided any evidence in favour of supersymmetry, pushing its limits beyond the TeV scale. Supersymmetric or not the Standard Model is an incomplete theory as it does not include gravity.

String theory is our best candidate for a consistent theory of quantum gravity that includes the basic constituents of the SM. String phenomenology focuses on the construction and study of phenomenological features of string derived gauge models. These include extensions of the SM or GUTs that comprise the SM. The research in this field has yielded low energy effective models with realistic characteristics. However, until recently the relative investigation mainly restricted in supersymmetric models. The absence of experimental evidence in favour of SUSY motivates the extension of the quest for the SM in the domain of non-supersymmetric string vacua [ [U, 口, 目,

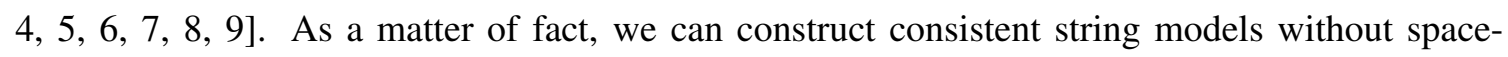
time supersymmetry. Ten-dimensional heterotic string theories comprise both the supersymmetric

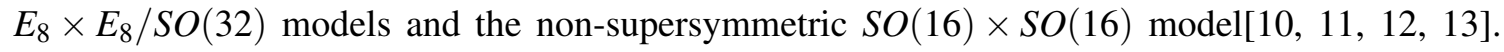
Even more interestingly, SUSY can be broken spontaneously at the string theory level. The ScherkSchwartz mechanism provides a paradigm of such breaking in the context of closed strings [प14, [5], ㅁ, 미, [8, [9]. In its simplest form the mechanism involves one of the extra dimensions, $X^{5}$, and a symmetry operator $Q$. Compactification in a circle of radius $R$ upon the action of $Q$, $\Phi\left(X^{5}+2 \pi R\right)=e^{i Q} \Phi\left(X^{5}\right)$, leads to a shifted tower of Kaluza-Kelin states for a charged field $\Phi$ in the theory. The mass shift is inversely proportional to the radius $R, M_{s}=Q / 2 \pi R$. Identifying $Q$ with the fermion number we obtain a split between fermionic and bosonic states belonging to the same multiplet leading to a SUSY breaking at a scale proportional to $m_{3 / 2} \sim 1 / R$.

The construction of realistic non-supersymmetric models, however, is hampered by some serious problems that arise due to the absence of SUSY. First, the cosmological constant is not guaranteed to vanish leading to dilaton tadpoles that destabilise the vacuum. Second, physical tachyons arise in special points of the moduli space. These issues need to be addressed in conjunction with the usual phenomenological requirements as the presence of chiral fermions and scalar Higgs doublets in the massless string spectrum.

In this article we summarise some recent work in the study of heterotic string models with SUSY broken spontaneously via the Scherk-Schwartz mechanism [20, [2], [2]]. In section 2 we define the class of semi-realistic models under investigation. We introduce a minimal set of phenomenological requirements and present the results of an initial computer search for models that meet these criteria. In section 3 we examine the cosmological constant and its dependence on the compactification moduli. We formulate a set of additional requirements and discuss the results of a full computer search for compactifications that fulfil all the constraints. In section 4 we put forward our conclusions. 


\section{A class of non-supersymmetric heterotic string models and phenomenological criteria}

For the purposes of our analysis we will consider a class of heterotic string models in the Free Fermionic Formulation (FFF) [23, 24]]. This framework is convenient for the study of the spectra as we can utilise the classification formalism developed in [25, [26, [27, [29, [30, [1], 132]. We will focus on a semi-realistic set of models with gauge symmetry $\mathrm{SO}(10) \times \mathrm{SO}(8)^{2} \times \mathrm{U}(1)^{9}$, where the $\mathrm{SO}(10)$ factor is assumed to be the "observable" gauge group. In the FFF this class of models is defined by a fixed set of basis vectors $\left\{\beta_{1}, \ldots, \beta_{9}\right\}$,

$$
\begin{aligned}
& \beta_{1}=\mathbf{1}=\left\{\psi^{\mu}, \chi^{1, \ldots, 6}, y^{1, \ldots, 6}, \omega^{1, \ldots, 6} \mid \bar{y}^{1, \ldots, 6}, \bar{\omega}^{1, \ldots, 6}, \bar{\eta}^{1,2,3}, \bar{\psi}^{1, \ldots, 5}, \bar{\phi}^{1, \ldots, 8}\right\} \\
& \beta_{2}=S=\left\{\psi^{\mu}, \chi^{1, \ldots, 6}\right\} \\
& \beta_{3}=T_{1}=\left\{y^{12}, \omega^{12} \mid \bar{y}^{12}, \bar{\omega}^{12}\right\} \\
& \beta_{4}=T_{2}=\left\{y^{34}, \omega^{34} \mid \bar{y}^{34}, \bar{\omega}^{34}\right\} \\
& \beta_{5}=T_{3}=\left\{y^{56}, \omega^{56} \mid \bar{y}^{56}, \bar{\omega}^{56}\right\} \\
& \beta_{6}=b_{1}=\left\{\chi^{34}, \chi^{56}, y^{34}, y^{56} \mid \bar{y}^{34}, \bar{y}^{56}, \bar{\psi}^{1, \ldots, 5}, \bar{\eta}^{1}\right\} \\
& \beta_{7}=b_{2}=\left\{\chi^{12}, \chi^{56}, y^{12}, y^{56} \mid \bar{y}^{12}, \bar{y}^{56}, \bar{\psi}^{1, \ldots, 5}, \bar{\eta}^{2}\right\} \\
& \beta_{8}=z_{1}=\left\{\bar{\phi}^{1, \ldots, 4}\right\} \\
& \beta_{9}=z_{2}=\left\{\bar{\phi}^{5, \ldots, 8}\right\}
\end{aligned}
$$

and a variable set of phases $c\left[\begin{array}{l}\beta_{i} \\ \beta_{j}\end{array}\right], i, j=1, \ldots, 9$ associated with generalised GSO projections (GGSO). It comprises $2^{9(9-1) / 2}+1 \sim 10^{11}$ in principle distinct configurations.

We will concentrate on the main phenomenological characteristics of models in the class. The first issue that needs to be addressed is the presence of tachyonic states. They can arise from the sectors $p T_{i}+q z_{1}+r z_{2}$ with $i=1,2,3$ and $p, q, r \in\{0,1\}$. Nevertheless, by projecting onto these states with phase dependent GGSO the tachyons can be eliminated. Therefore, our first phenomenological criterion to select amongst models between the aforementioned configurations is related to the absence of tachyons. To this end, we derived analytic, albeit lengthy, formulae regarding tachyonic state number that we incorporate in our model search.

Next we consider the chirality of fermionic states transforming in the spinorials of the "observable" $\mathrm{SO}(10)$ gauge group factor, that is the number of spinorials (16) minus the number of antisponorials $(\overline{\mathbf{1 6}})$. These states arise from the twisted sectors $B_{p q}^{I}=S+b_{p q}^{I}, I=1,2,3$ with $b_{p q}^{1}=b^{1}+p T_{2}+q T_{3}, b_{p q}^{2}=b^{2}+p T_{1}+q T_{3}, b_{p q}^{3}=x+b^{1}+b^{2}+p T_{1}+q T_{2}, p, q \in\{0,1\}$, and $x=\mathbf{1}+S+\sum_{i=1}^{3} T_{i}+\sum_{k=1}^{2} z_{k}$. In terms of the GGSO phases the net chirality, to be identified with the net number of fermion generations is

$$
N_{f}=-4 \sum_{I=1,2,3} \sum_{p, q=0,1} P_{p q}^{I} c\left[\begin{array}{c}
B_{p q}^{I} \\
R^{I}
\end{array}\right]
$$

where $R^{1}=R^{2}=S+b_{2}+(1-q) T_{3}, R^{3}=S+b_{1}+(1-q) T_{1}$ and

$$
P_{p q}^{I}=\frac{1}{2^{3}}\left(1-c\left[\begin{array}{c}
B_{p q}^{I} \\
T_{I}
\end{array}\right]\right)\left(1-c\left[\begin{array}{c}
B_{p q}^{I} \\
z_{1}
\end{array}\right]\right)\left(1-c\left[\begin{array}{c}
B_{p q}^{I} \\
z_{2}
\end{array}\right]\right) .
$$


That said, the second phenomenological model selection criterion is the chiral spectrum, that is $N_{f} \neq 0$.

Next, we turn to the number of scalar $\mathrm{SO}(10)$ vectorials $(\mathbf{1 0})$ that are also of phenomenological importance as they accommodate the SM breaking Higgs doublets. These states arise from both the untwisted and the twisted sector. It turns out that the total number of $\mathrm{SO}(10) \mathrm{s}$ is model dependent, however, all models in this class are endowed with three vectorial pairs arising from the untwisted sector. As a result, no additional constraint arises from this requirement.

The implementation of the Scherks-Schwartz SUSY breaking mechanism turns out to be very delicate in the fermionic formulation. Actually, many of the models in the class under consideration correspond to explicitly broken SUSY models and should be rejected. A careful analysis reveals a set of additional intricate constraints.

Having formulated a minimal set of phenomenological requirements we now turn to the quest of models satisfying these constraints. A comprehensive computer scan over the full parameter space leads to 76552 models that are compatible with all phenomenological criteria. Detailed results are presented in figure $\mathbb{W}$ where we plot the number of acceptable models versus the net chirality $\left(N_{f}\right)$. Although derived here by assuming a semi-realistic framework these results are rather non-trivial, as the selection criteria become very stringent when applied simultaneously.

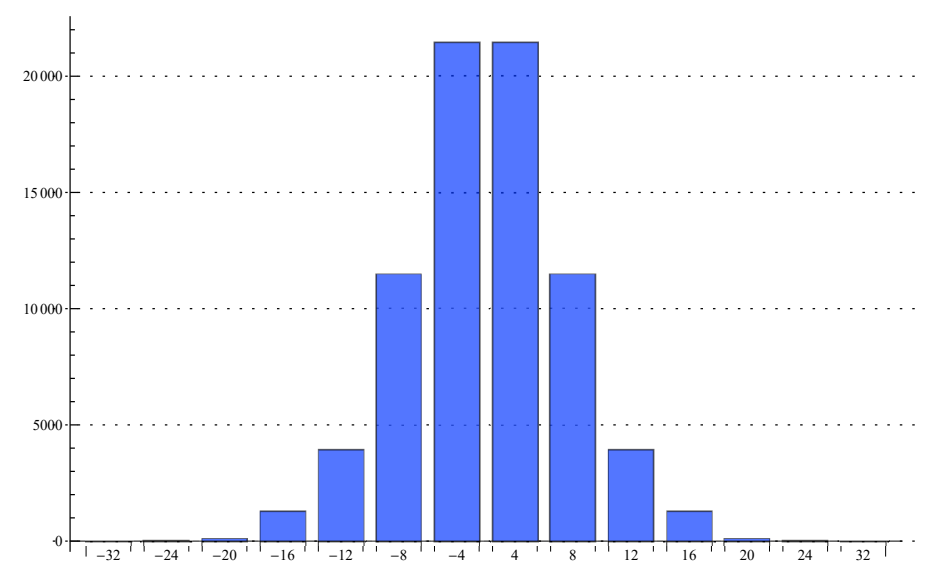

Figure 1: Number of acceptable models versus net chirality $\left(N_{f}\right)$ in a class of $10^{11}$ configurations.

\section{One-loop potentials and cosmological constant}

In the context of the Scherk-Schwarz SUSY breaking the tree-level scalar potential being of the no-scale type [33] vanishes at the minimum resulting in vanishing vacuum energy and massless moduli. However, at one-loop level the scalar potential receives not trivial corrections of the form

$$
V_{\text {one-loop }}\left(t_{I}\right)=-\frac{1}{2(2 \pi)^{4}} \int_{\mathscr{F}} \frac{d^{2} \tau}{\tau_{2}^{3}} Z\left(\tau, \bar{\tau} ; t_{I}\right),
$$

where $t_{I}=\left\{T^{(I)}, U^{(I)}, I=1,2,3\right\}$ are the Kähler and complex structure moduli of three 2-tori, $Z\left(\tau, \bar{\tau} ; t_{I}\right)$ is the string partition function and the integration extends over a fundamental domain $\mathscr{F}$ of the worldsheet torus. The computation of the vacuum energy as a function of the moduli 
is a very tedious task even numerically. It can be simplified by assuming that the supersymmetry breaking is realised utilising the first torus while the other two torii are fixed at the fermionic point. A systematic analysis shows that for $t_{I} \sim 1, I=1,2$ the cosmological constant takes big values not compatible with the observational data. However, small values can arise in large volume compactifications. This is of interest, since it is compatible with low energy supersymmetry breaking. Parametrising the first torus by $T=T^{(1)}=T_{1}+i T_{2}, U=U^{(1)}=U_{1}+i U_{2}$ we can obtain the asymptotic expression [20]

$$
\lim _{T_{2} \gg 1} V_{\text {one-loop }}(T, U)=-\frac{\left(n_{B}-n_{F}\right)}{2^{4} \pi^{7} T_{2}^{2}} \sum_{m_{1}, m_{2} \in \mathbb{Z}} \frac{U_{2}^{3}}{\left|m_{1}+\frac{1}{2}+U m_{2}\right|^{6}}+\mathscr{O}\left(e^{-\pi \sqrt{2 T_{2}}}\right)
$$

In addition, the gravitino mass reads

$$
m_{3 / 2}=\frac{|U|}{\sqrt{T_{2} U_{2}}}
$$

For a square torus, $T=i R_{1} R_{2}$ and $U=i R_{2} / R_{1}$, this reduces to $m_{3 / 2}=1 / R_{1}$. Assuming for example $R_{1}=R_{2}=R=\sqrt{T_{2}}$ we get $m_{3 / 2}=1 / R=1 / \sqrt{T_{2}}$ and

$$
V_{\text {one-loop }}(T, U)=-\frac{\left(n_{B}-n_{F}\right)}{2^{4} \pi^{7}} \xi(U) m_{3 / 2}^{2}+\text { exponentially suppressed terms }
$$

where $\xi(U)$ is a function of $U$ defined in (B.2). It is clear that the cosmological constant is suppressed at the limit $T_{2} \gg 1$, however, in a generic model the obtained values are not sufficiently small even if we lower the SUSY breaking scale $m_{3 / 2}$ down to a few TeV.

From the previous discussion, one concludes that an interesting exception arises in the case of nonsupersymmetric models with boson-fermion degeneracy $n_{B}=n_{F}$ also referred as "super no-scale" models [B4, [35, B36, B37, B8, B9, ,40]. The leading term in (B.2) vanishes resulting in exponentially suppressed cosmological constant for $T_{2} \gg 1$. This scenario is of particular phenomenological interest, however, one needs to investigate the compatibility with other phenomenological constraints discussed in Section 2 (e.g. fermion chirality).

Another issue under investigation is the sign of the cosmological constant. Recent observations indicating a tiny but positive cosmological constant seem to be in contradiction with some no-go theorems regarding the existence of de-Sitter vacua in string theory [4]]. It is thus interesting to check the sign of the cosmological constant in the models under consideration. To this end, we computed the the vacuum energy at the fermionic point and utilised its sign as an additional criterion in the model classification.

Combining the phenomenologigal criteria of the previous section with the aforementioned criteria associated to the cosmological constant in a comprehensive and tedious computer search we find 1792 models that meet all requirements. In firure $\square$ we plot the number of acceptable models as a function of the net fermion chirality $\left(N_{f}\right)$.

For the models that comply with the requirements we also studied the shape of the one loop potential as a function of the moduli. The dependence on the $T_{2}$ modulus is presented in figure [ $]$ for two typical models. Model (a) exhibits a single maximum at the fermionic point while model (b) has a local minimum at the fermionic point and local maxima laying astride the minimum. This behaviour is compatible with a scenario where the unstable or meta-stable potential drives the modulus towards large volume and leads (once stabilised [42]) to low energy SUSY breaking with exponentially suppressed cosmological constant. 


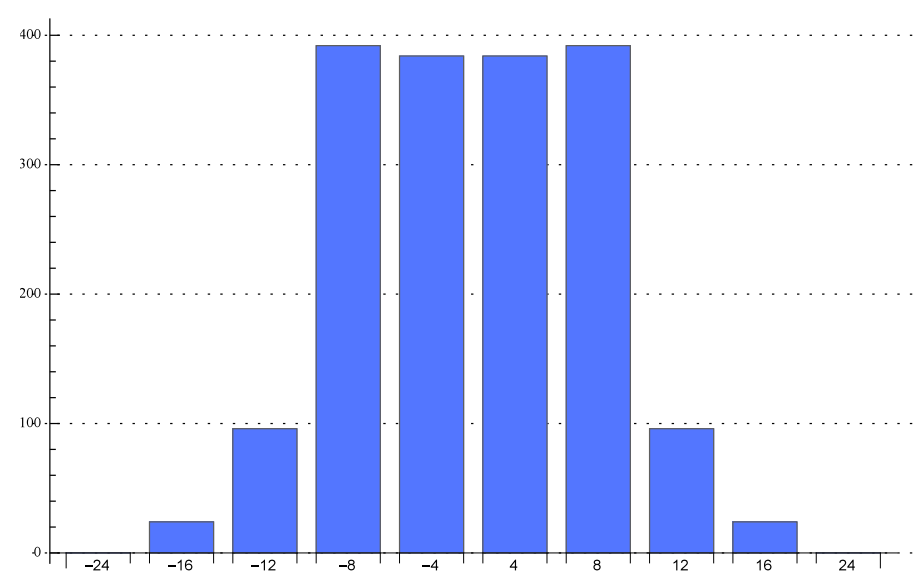

Figure 2: Number of acceptable models with fermion-boson degeneracy and positive cosmological constant versus net chirality $\left(N_{f}\right)$ in a class of $10^{11}$ configurations.
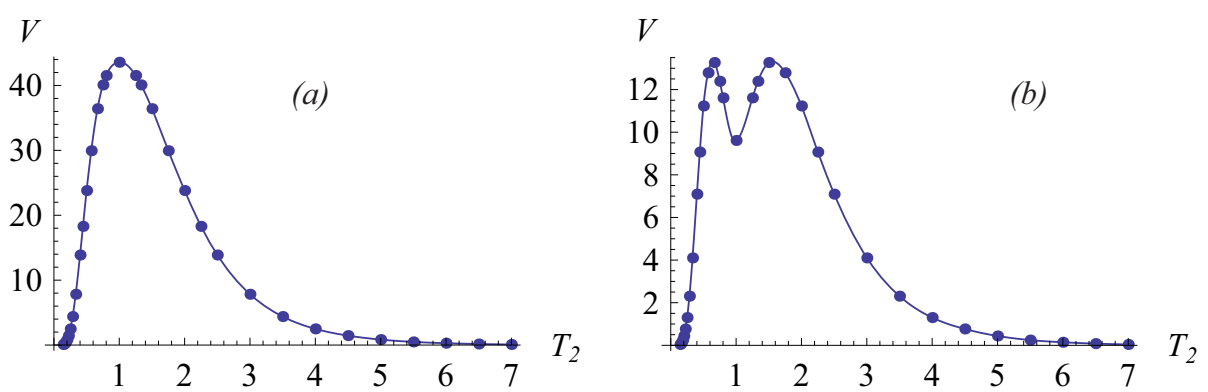

Figure 3: Plots of the one loop potential as a function of the $T_{2}$ modulus for two typical models satisfying all requirements.

\section{Conclusions}

The lack of experimental evidence supporting SUSY motivates the study of gauge models resulting from non-supersymmetric string compactifications. However, abandoning space-time supersymmetry represents an important challenge for string phenomenology. The absence of tachyons in the string spectrum is not automatic and has to be examined on a model-by-model basis. Moreover, the vacuum energy no longer vanishes identically and leads to large dilaton tadpoles that destabilise the vacuum of the theory.

In this work, we investigated a big class of heterotic string compactifications where SUSY is broken via the Scherk-Schwatz mechanism and identified a subclass of models with interesting phenomenological properties. A basic characteristic of these models is the fermion-boson degeneracy $n_{B}=n_{F}$ that guarantees the exponential suppression of the cosmological constant at large volume. Moreover, these models possess chiral fermions accommodated in the spinorial representation of the observable gauge group. In addition, the models under consideration exhibit positive cosmological constant at the string scale and an unstable/meta-stable potential that could drive dynamically the moduli towards large volumes triggering SUSY breaking at low energies.

An additional issue we have not addressed here is the so called decompactification problem that is the linear growth of the gauge couplings with the compactification volume [43], 44, 45], 46, 
47, 48, 49, 101, [1], 52]. Possible solutions to the decompactification problem have been proposed in [2], [53] .

All-together, these developments shed light to the non-supersymmetric side of the string landscape and encourage us to explore in detail the phenomenology of non-supersymmetric string models with more realistic gauge groups.

\section{Acknowledgments}

J. R. would like to thank the organizers of Corfu Summer Institute 2019 "School and Workshops on Elementary Particle Physics and Gravity" (CORFU2019) for their hospitality. 


\section{References}

[1] B. Gato-Rivera and A. N. Schellekens, Phys. Lett. B 656 (2007) 127 doi:10.1016/j.physletb.2007.09.009 [arXiv:0709.1426 [hep-th]]; Phys. Lett. B 671 (2009) 105 doi:10.1016/j.physletb.2008.11.039 [arXiv:0810.2267 [hep-th]].

[2] M. Blaszczyk, S. Groot Nibbelink, O. Loukas and S. Ramos-Sanchez, JHEP 1410 (2014) 119 doi:10.1007/JHEP10(2014)119 [arXiv:1407.6362 [hep-th]].

[3] S. Abel, K. R. Dienes and E. Mavroudi, Phys. Rev. D 91 (2015) no.12, 126014 doi:10.1103/PhysRevD.91.126014. [arXiv:1502.03087 [hep-th]].

[4] M. Blaszczyk, S. Groot Nibbelink, O. Loukas and F. Ruehle, JHEP 1510 (2015) 166 doi:10.1007/JHEP10(2015)166 [arXiv:1507.06147 [hep-th]].

[5] S. Groot Nibbelink, O. Loukas and F. Ruehle, Fortsch. Phys. 63 (2015) 609 doi:10.1002/prop.201500041 [arXiv:1507.07559 [hep-th]].

[6] J. M. Ashfaque, P. Athanasopoulos, A. E. Faraggi and H. Sonmez, Eur. Phys. J. C 76 (2016) no.4, 208 doi:10.1140/epjc/s10052-016-4056-2. [arXiv:1506.03114 [hep-th]].

[7] S. Abel, K. R. Dienes and E. Mavroudi, Phys. Rev. D 97, no. 12, 126017 (2018) doi:10.1103/PhysRevD.97.126017 [arXiv:1712.06894 [hep-ph]].

[8] A. E. Faraggi, V. G. Matyas and B. Percival, Eur. Phys. J. C 80 (2020) no.4, 337 doi:10.1140/epjc/s10052-020-7894-x [arXiv:1912.00061 [hep-th]].

[9] M. McGuigan, [arXiv:1907.01944 [hep-th]].

[10] D. J. Gross, J. A. Harvey, E. J. Martinec and R. Rohm, Phys. Rev. Lett. 54, 502 (1985). doi:10.1103/PhysRevLett.54.502

[11] L. Alvarez-Gaume, P. H. Ginsparg, G. W. Moore and C. Vafa, Phys. Lett. B 171, 155 (1986). doi:10.1016/0370-2693(86)91524-8

[12] L. J. Dixon and J. A. Harvey, Nucl. Phys. B 274, 93 (1986). doi:10.1016/0550-3213(86)90619-X

[13] H. Kawai, D. C. Lewellen and S. H. H. Tye, Phys. Rev. D 34, 3794 (1986). doi:10.1103/PhysRevD.34.3794

[14] R. Rohm, Nucl. Phys. B 237 (1984) 553. doi:10.1016/0550-3213(84)90007-5

[15] C. Kounnas and M. Porrati, Nucl. Phys. B 310355 (1988). doi:10.1016/0550-3213(88)90153-8.

[16] S. Ferrara, C. Kounnas, M. Porrati and F. Zwirner, Nucl. Phys. B 31875 (1989) . doi:10.1016/0550-3213(89)90048-5

[17] C. Kounnas and B. Rostand, Nucl. Phys. B 341641 (1990) . doi:10.1016/0550-3213(90)90543-M.

[18] J. Scherk and J. H. Schwarz, Phys. Lett. B 8260 (1979). doi:10.1016/0370-2693(79)90425-8.

[19] J. Scherk and J. H. Schwarz, Nucl. Phys. B 15361 (1979) . doi:10.1016/0550-3213(79)90592-3.

[20] I. Florakis and J. Rizos, Nucl. Phys. B 913, 495 (2016) doi:10.1016/ j.nuclphysb.2016.09.018 [arXiv:1608.04582 [hep-th]].

[21] I. Florakis and J. Rizos, Nucl. Phys. B 921, 1 (2017) doi:10.1016/ j.nuclphysb.2017.05.002 [arXiv:1703.09272 [hep-th]].

[22] J. Rizos, Int. J. Mod. Phys. A 33 (2018) no.34, 1845002 doi:10.1142/S0217751X18450021 
[23] I. Antoniadis, C. P. Bachas and C. Kounnas, Nucl. Phys. B 289, 87 (1987). doi:10.1016/0550-3213(87)90372-5.

[24] I. Antoniadis and C. Bachas, Nucl. Phys. B 298, 586 (1988). doi:10.1016/0550-3213(88)90355-0.

[25] A. Gregori, C. Kounnas and J. Rizos, A. Gregori, C. Kounnas and J. Rizos, Nucl. Phys. B 549, 16 (1999) doi:10.1016/S0550-3213(99)00135-2 [hep-th/9901123].

[26] A. E. Faraggi, C. Kounnas, S. E. M. Nooij and J. Rizos, Nucl. Phys. B 695, 41 (2004) doi:10.1016/j.nuclphysb.2004.06.030 [hep-th/0403058].

[27] A. E. Faraggi, C. Kounnas and J. Rizos, Phys. Lett. B 648, 84 (2007) doi:10.1016/j.physletb.2006.09.071 [hep-th/0606144].

[28] B. Assel, K. Christodoulides, A. E. Faraggi, C. Kounnas and J. Rizos, Nucl. Phys. B 844, 365 (2011) doi:10.1016/j.nuclphysb.2010.11.011 [arXiv:1007.2268 [hep-th]].

[29] A. Faraggi, J. Rizos and H. Sonmez, Nucl. Phys. B 886, 202 (2014) doi:10.1016/j.nuclphysb.2014.06.025 [arXiv:1403.4107 [hep-ph]].

[30] A. E. Faraggi, J. Rizos and H. Sonmez, Nucl. Phys. B 927, 1 (2018) doi:10.1016/j.nuclphysb.2017.12.006 [arXiv:1709.08229 [hep-th]].

[31] A. E. Faraggi, G. Harries and J. Rizos, Nucl. Phys. B 936 (2018), 472-500 doi:10.1016/j.nuclphysb.2018.09.028 [arXiv:1806.04434 [hep-th]].

[32] A. E. Faraggi, G. Harries, B. Percival and J. Rizos, Nucl. Phys. B 953 (2020), 114969 doi:10.1016/j.nuclphysb.2020.114969 [arXiv:1912.04768 [hep-th]].

[33] E. Cremmer, S. Ferrara, C. Kounnas and D. V. Nanopoulos, Phys. Lett. B 133 (1983) 61 doi:10.1016/0370-2693(83)90106-5

[34] C. Kounnas and H. Partouche, Nucl. Phys. B 913, 593 (2016) doi:10.1016/j.nuclphysb.2016.10.001 [arXiv:1607.01767 [hep-th]].

[35] T. Coudarchet and H. Partouche, Nucl. Phys. B 933, 134 (2018) doi:10.1016/j.nuclphysb.2018.06.009 [arXiv:1804.00466 [hep-th]].

[36] T. Coudarchet, C. Fleming and H. Partouche, Nucl. Phys. B 930, 235 (2018) doi:10.1016/j.nuclphysb.2018.03.002 [arXiv:1711.09122 [hep-th]].

[37] B. Aaronson, S. Abel and E. Mavroudi, Phys. Rev. D 95, no. 10, 106001 (2017) doi:10.1103/PhysRevD.95.106001 [arXiv:1612.05742 [hep-th]].

[38] S. Abel and R. J. Stewart, Phys. Rev. D 96, no. 10, 106013 (2017) doi:10.1103/PhysRevD.96.106013 [arXiv:1701.06629 [hep-th]].

[39] H. Itoyama and S. Nakajima, [arXiv:2003.11217 [hep-th]].

[40] S. Abel, T. Coudarchet and H. Partouche, [arXiv:2003.02545 [hep-th]].

[41] J. M. Maldacena and C. Nunez, Int. J. Mod. Phys. A 16 (2001), 822-855 doi:10.1142/S0217751X01003937 [arXiv:hep-th/0007018 [hep-th]].

[42] S. Abel, JHEP 1611, 085 (2016) doi:10.1007/JHEP11(2016)085 [arXiv:1609.01311 [hep-th]].

[43] I. Antoniadis, Phys. Lett. B 246, 377 (1990). doi:10.1016/0370-2693(90)90617-F

[44] E. Kiritsis, C. Kounnas, P. M. Petropoulos and J. Rizos, Phys. Lett. B 385, 87 (1996) doi:10.1016/0370-2693(96)00880-5 [hep-th/9606087]. 
[45] P. M. Petropoulos and J. Rizos, Phys. Lett. B 374, 49 (1996) doi:10.1016/0370-2693(96)00230-4 [hep-th/9601037].

[46] E. Kiritsis, C. Kounnas, P. M. Petropoulos and J. Rizos, Nucl. Phys. B 540, 87 (1999) doi:10.1016/S0550-3213(98)00713-5 [hep-th/9807067].

[47] C. P. Bachas, JHEP 9811, 023 (1998) doi:10.1088/1126-6708/1998/11/023 [hep-ph/9807415].

[48] I. Antoniadis and C. Bachas, Phys. Lett. B 450, 83 (1999) doi:10.1016/S0370-2693(99)00102-1 [hep-th/9812093].

[49] A. E. Faraggi, C. Kounnas and H. Partouche, Nucl. Phys. B 899, 328 (2015) doi:10.1016/j.nuclphysb.2015.08.001 [arXiv:1410.6147 [hep-th]].

[50] C. Angelantonj, I. Florakis and M. Tsulaia, Phys. Lett. B 736, 365 (2014) doi:10.1016/j.physletb.2014.08.001 [arXiv:1407.8023 [hep-th]].

[51] C. Angelantonj, I. Florakis and M. Tsulaia, Nucl. Phys. B 900, 170 (2015) doi:10.1016/j.nuclphysb.2015.09.007 [arXiv:1509.00027 [hep-th]].

[52] C. Angelantonj, I. Florakis and B. Pioline, Nucl. Phys. B 897781 (2015) doi:10.1016/j.nuclphysb.2015.06.009 [arXiv:1502.00007 [hep-th]].

[53] C. Angelantonj and I. Florakis, Phys. Lett. B 789 (2019), 496-501 doi:10.1016/j.physletb.2018.12.054 [arXiv:1812.06915 [hep-th]]. 